\title{
Aldehyde dehydrogenase- 1 expression and prognosis in triple-negative breast cancer
}

\author{
Edvin Murrja ${ }^{1}$, Zeynep Hande Turna ${ }^{2}$, Mehmet Akif Ozturk ${ }^{2}$, Ahmet Cinkaya ${ }^{3}$, Tulin Ozturk ${ }^{4}$
}

\section{Abstract}

Objective: Understanding of other biological characteristics of Triple-negative breast cancer, the development of new therapeutical approaches and identification of new markers is necessary due to the lack of biological markers like ER, PR and HER2. The purpose of this study was to investigate the prognostic effect of the expression of aldehyde dehydrogenase-1 (ALDH1) in TNBC and its relationship with the clinicopathological features

Methods: In this study 87 patient files were searched for clinico-pathological data obtained from the files and paraffin blocks. The prognostic value of these clinical data and ALDH1 positivity were evaluated by determining disease-free survival.

Results: TNM stage I vs III $(p=0.03)$, vascular invasion $(p=0.05)$, chemotherapy indication $(p=0.02)$ were significantly associated with DFS. Multivariable analyses didn't demonstrate any statistically significant relationship between ALDH1 $(\mathrm{p}=0.61)$ and DFS.

Conclusions: We didn't find any statistically significant relationship between ALDH1 positivity and DFS.

There was no correlation between ALDH1 expression and tumor's pathological features.

Keywords: aldehyde dehydrogenase 1, immunohistochemistry, triple-negative breast cancer

\section{Introduction}

Triple-negative breast cancer is defined by absent expression of the estrogen receptor [ER], progesterone receptor $[\mathrm{PR}]$, and the human epidermal growth factor receptor 2 [HER2] negative status [1]. Given the lack of these biologic markers endocrine therapy and HER2-directed therapies are not recommended in the treatment of TNBC [1820].Although the same general principles used in the diagnosis and treatment of non-triple-negative breast cancer generally apply to TNBC, it has a lot of differences in risk factors, molecular and histologic features, clinical behavior and chemotherapy sensitivity [2-6].TNBC defines a heterogeneous mix of breast tumors [7]. TNBC generally has a poorer prognosis among patients with breast cancer [21]. ER, PR and HER2 are the important biologic markers used in targeted treatment of breast cancer [22]. Understanding of other biological characteristics of TNBC, the development of new therapeutical approaches and identification of new markers is necessary due to the lack of biological markers like ER, PR and HER2.CD44+, CD24-/low and ALDH1+ are the most consistently used biomarkers to identify the breast cancer stem cell groups [16-17].The clinical importance of ALDH1 in TNBC is a less investigated issue. The purpose of this study was to investigate the prognostic effect of the expression of aldehyde dehydrogenase-1 (ALDH1) in TNBC and its relationship with the clinico-pathological features

Received: 15 Aug. 2014, Revised 20 Aug. 2014, Accepted 24 Aug. 2014, Available Online 30 Aug. 2014

${ }^{1}$ Istanbul University, Faculty of Medicine, Department of Internal Medicine, Istanbul, Turkey

2 İstanbul University, Faculty of Medicine, Department of Medical Oncology, Istanbul, Turkey

${ }^{3}$ Celal Bayar University, Faculty of Medicine, Department of Radiation Oncology, Manisa Turkey

${ }^{4}$ Istanbul University, Faculty of Medicine, Department of Pathology, İstanbul, Turkey

*Corresponding Author: Edvin Murrja E-mail: dredvin@yahoo.com 


\section{Materials and Methods}

In this study we included 87 patients followed between 2000 and 2012 in Cerrahpaşa Faculty of Medicine, Department of Medical Oncology Clinic diagnosed with TNBC. Formalin-fixed, paraffinembedded tissues of these patients were retrieved from the Department of Pathology, in the same hospital.

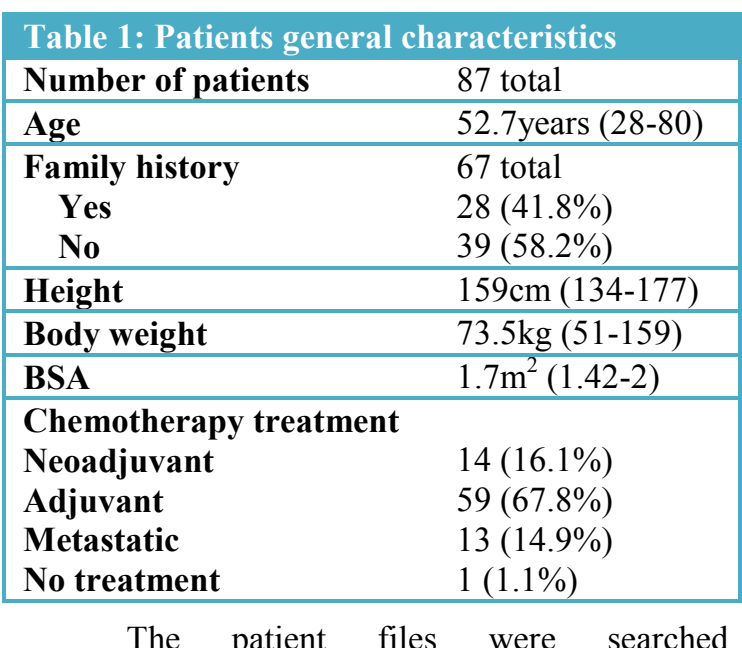

retrospectively for age, sex, comorbidities, the presence of family history of cancer, breast cancer detection methods, pregnancy/number of children, height, weight, BSA, ECOG, biopsy date, whether or not operated, type of surgery, tumor size / TNM stage and localization, axillary nodal status, the presence of the tumor in the border of surgery, pathologic evaluation, the first chemotherapy option: adjuvant, neoadjuvant or metastatic, chemotherapy regimen series, number of cycles, the start/end dates and all were recorded.

The surveillance after treatment for early stage breast cancer was performed each 3 months for the first two years, every 6 months for the next three years and then every year after five years. Patients with metastatic disease were followed with a surveillance routine after receiving 3 and 6 cycles of chemotherapy.

Formalin-fixed, paraffin-embedded tissues was cut into $3 \mu \mathrm{m}$ thick sections, deparaffinided, stained with hematoxylin-eosin and

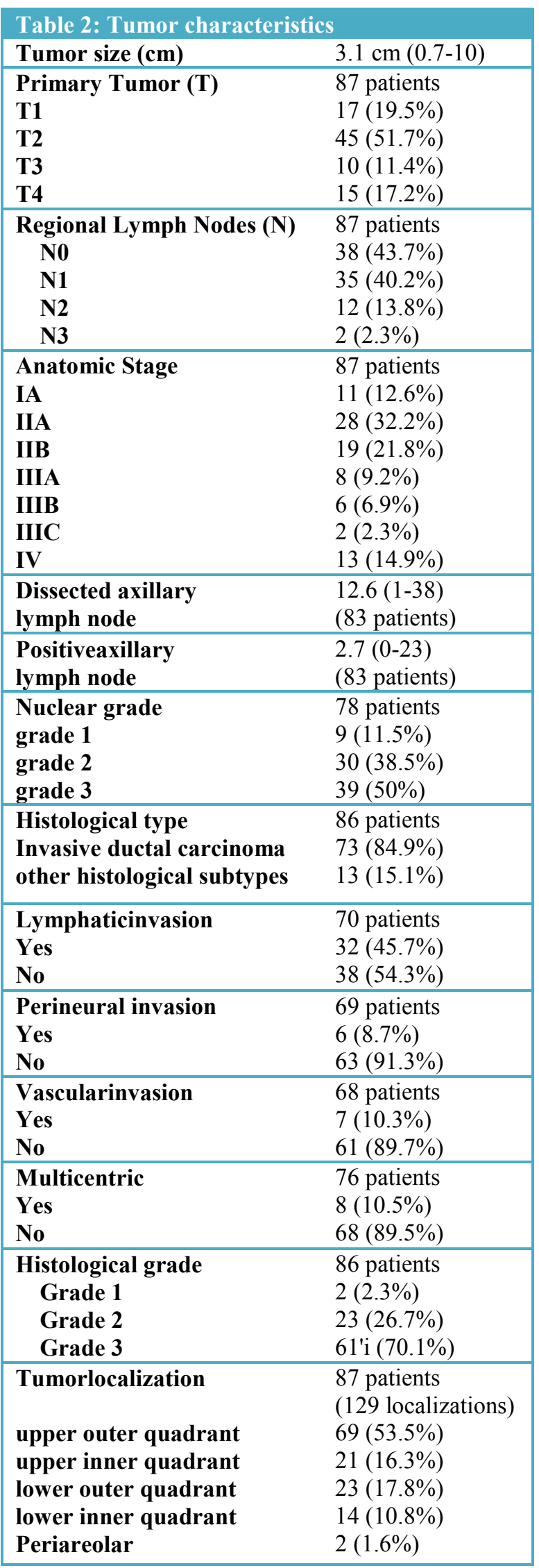


immunohistochemically with ALDH1 (ALDH1a1: Biocare assay kit) then examined by light microscopy.

The prognostic value of these clinical data and ALDH1 positivity were evaluated by determining disease-free survival and tumor characteristics from the clinicopathological data obtained from the files and paraffin blocks.

Immunohistochemical staining ofALDH1 was classified as positive when tumor cells showed cytoplasmic positivity and negative when tumor cells showed cytoplasmic negativity.

The prognostic value of these clinical data and ALDH1 positivity were evaluated by determining disease-free survival and tumor characteristics from the clinicopathological data obtained from the files and paraffin blocks. Among 87 files included in the present study, the metastatic ones and those who received neoadjuvant therapy but found to be metastatic before receiving adjuvant therapy were excluded. Statistical analyses include 71 patients.
The DFS (disease-free survival) was defined as the time from the diagnosis to the date of breast-cancerderived relapse/metastasis.

DFS for patients who received adjuvant therapy (month): (the date of relapse or last visit date for those without relapse -operation date)/30, for those who received neoadjuvant therapy (month): (the date of relapse or last visit date for those without relapse - operation date after neoadjuvant therapy) $/ 30$.

\section{Statistical Analaysis}

Statistical analysis was performed using the SPSS (SPSS 16.0, SPSS Inc. Chicago, Illinois). DFS was calculated with the Kaplan-Meier technique, analyses of the prognostic factors and DFS were performed with the log-rank test. Multivariable analyses were conducted for the factors statistically significant in the Cox Proportional Hazards model.

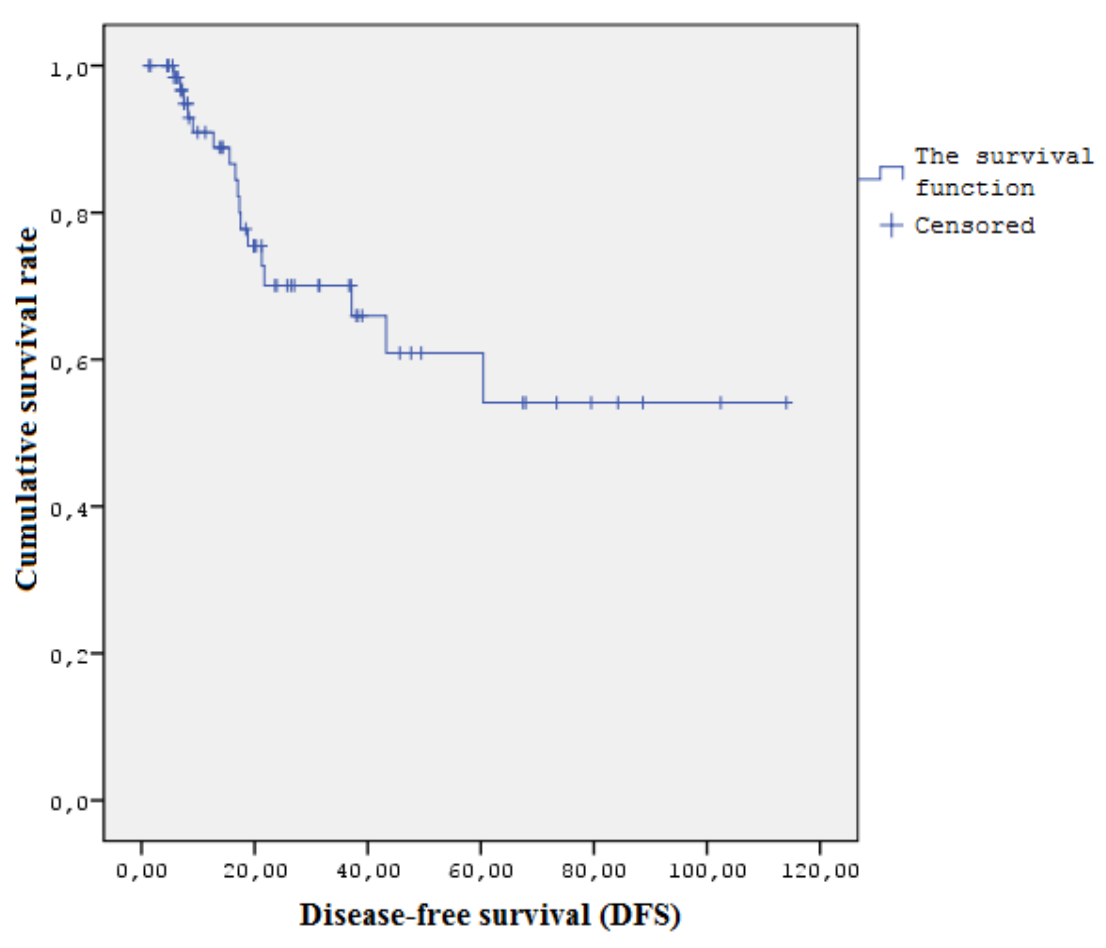

Figure 1: DFS for non-metastatic group 


\section{Results}

Study population: The age on the patient files included in the present study ranged from 28 to 80 years old (mean of 52.7 years). 62 patients had no comorbidity, 5 had diabetes mellitus, 17 had essential hypertension, and 2 had hypothyroidism. The family history was not noted in 20 percent of the patient files. $39 \%$ of the patients had no family history, $28 \%$ of them had cancer diagnosed in their family. Based on the diagnostic evaluation of suspected breast cancer one of the patients was diagnosed after physical examination, one during routine screening and all others (n: 85) were suspected by their self-examination. Height ranged from 134 to $177 \mathrm{~cm}$ (mean of $159 \mathrm{~cm}$ ), body weight 51 to $159 \mathrm{~kg}$ (mean of $73.5 \mathrm{~kg}$ ), Body Surface Area (BSA) ranged from 1.42 to $2 \mathrm{~m}^{2}$ (mean of $1.7 \mathrm{~m}^{2}$ ).

Tumor characteristics: Among all the patients 13 (14.9\%) were metastatic at the beginning, 14 $(16.1 \%)$ received neoadjuvant treatment, 59 received adjuvant treatment, 1 (1.1\%) didn't receive any treatment. Tumor size was mean of $3.1 \mathrm{~cm}$ $(0.7-10 \mathrm{~cm}) .17$ of the cases $(19.5 \%)$ were $\mathrm{Tl}$, 45(51.7\%) T2, 10 (11.4\%) T3, 15 (17.2\%) T4. 38 of the cases were N0, $35 \mathrm{N1}, 12 \mathrm{~N} 2,2 \mathrm{~N} 3$.

Of the 78 patients with known nuclear grade 9 were $(11.5 \%)$ grade $1,30(38.5 \%)$ grade 2 and $39(50 \%)$ grade 3 . According to the histologicalgrade $2.3 \%$ grade $1,26.7 \%$ grade 2 and $70.1 \%$ grade 3 . According to the anatomic stage 11 IA, 28 IIA, 19 IIB, 8 IIIA, 6 IIIB, 2 IIIC, 13 IV.

Of all patients included in the present study, 84.9\% of cases were invasive ductal carcinoma, $15.1 \%$ the other histological types.

$45.7 \%$ of the tumors showed lymphatic invasion whereas $54.3 \%$ showed no invasion. $8.7 \%$ of the tumors showed perineural invasion whereas $91.3 \%$ showed no invasion. $10.3 \%$ of the tumors showed vascular invasion whereas $89.7 \%$ showed no invasion. $10.5 \%$ of the tumors were multicentric whereas $89.5 \%$ were unicentric.Among 87 patients,

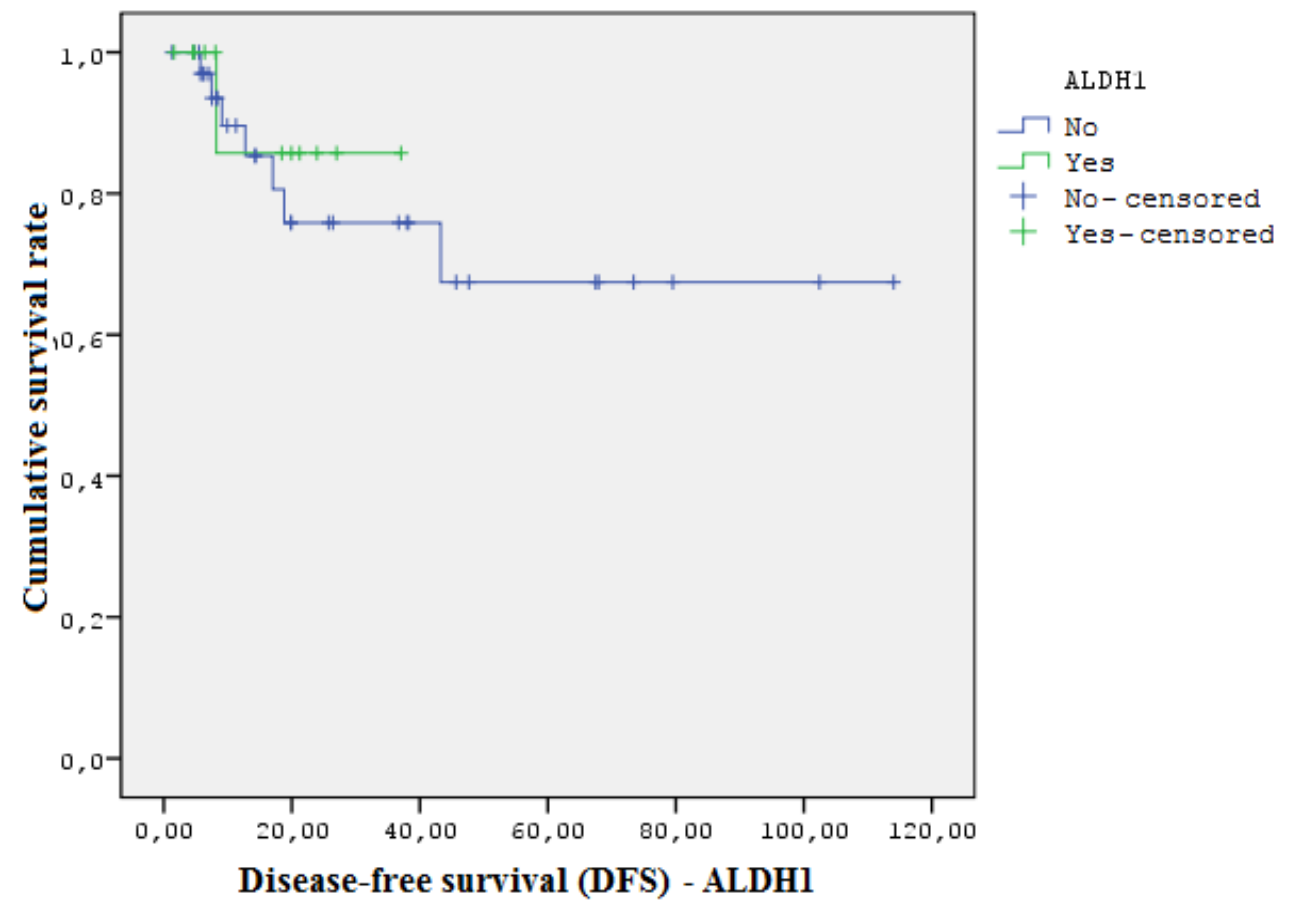

Figure 2: DFS according to ALDH1 expression 
51 had tumor on their right breast, 36 on their left breast. Of the 129 localizations seen (knowing than some of the tumors locate in more than one quadrant) $69(53.5 \%)$ were located upper outer, 21 (16.3\%)upper inner, 23 (17.8\%)lower outer, 14 (10.8\%)lower inner, 2 (1.6\%) periareolar.

Surgicaltreatment: Among 70 patients undergoing surgery,6 Mastectomy and Sentinel lymph node biopsy (SLNB), 23Breast-conserving surgery (BCS) and Axillary lymph node dissection (AD), 11 Breast-conserving surgery (BCS) and Sentinel lymph node biopsy (SLNB), 27 Modified radical mastectomy (MRM), 2 Roll excision, 1 Total mastectomywas performed.

Medicaltreatment: 87 patients included in this study received chemotherapy, 59 of them adjuvant (8 AC, 27 FEC/FAC, 6 FAC+Taxane, 11 AC+Taxane, $5 \mathrm{CMF}, 1$ Cyclophosphamide + Taxane), 14 neoadjuvant (10 FAC+Taxane, 1 FEC/FAC, 1 AC+Taxane, 1 Cyclophosphamide + Taxane, 1 Taxane), 13 metastaticdisease chemotherapy regiments, 1 patient didn't accept the treatment.

Disease-free survival (DFS) results: A total of 18 patients (\%25.4)had relapseduring the follow-up. The median follow-upperiod until relapse was 18.64 months $(0.4$ - 46.7). The median follow-up period for all patients was714 days (31-2645). DFS of non-metastaticgroup is shown on Figure 1. A total of 11 patients were found to be ALDH1positive. Analysis of the DFS showed no difference among ALDH1-positive and ALDH1-negative tumors $(\mathrm{p}=0.61)$ (Figure 2$)$

Multivariable analyses were conducted for the factors that were associated with DFS in the univariable analyses. Multivariable analyses didn't demonstrate any statistically significant relationship between ALDH1 $(\mathrm{p}=0.930)$, other prognostic factors and DFS. There was no correlation between
ALDH1 expression and tumor's pathological features.

\section{Discussion}

Breast cancer is a heterogeneous disease with a lot of histological special types showing different molecular, histologic and clinic features [15]. We achieved a significant reduction in breast cancer mortality with the screening programs and treatment facilities in adjuvant treatment of early stage breast cancer. The mortality rates are being reduced even more by the use of targeted therapy. TNBC accounts for $15 \%$ to $20 \%$ of breast cancers [3].Endocrine therapy and HER2-directed therapies are not used in the treatment of TNBC since it is characterized by the lack of biological markers like ER, PR and HER2[23]. TNBC generally has a poorer prognosis compared to patients with other breast cancer subtypes. Considerable effort has been made to develop new therapeutical approaches in TNBC [8].

The classical pathological variables such as tumor grade, lymph node status, and tumor size, are the most important prognostic factors in breast cancer.Ki67 status, tumor grade, lymphatic, perineural, vascular invasion are also independent prognostic factors that can affect the therapy choice $[9,11]$.

Based on the diagnostic evaluation of suspected breast cancer one of the patients was diagnosed after physical examination, one during routine screening and all others (n:85) were suspected by their self-examination. These findings support the fact that TNBC will more likely present as an 'interval cancer' between two screenings. These findings also show that TNBC screening and diagnosis need to be improved [12-14].

In our study 14 patients $(16.1 \%)$ received neoadjuvant therapy because of locally advanced disease, 59 patients $(67.8 \%)$ received adjuvant 
therapy. DFS of patients receiving neoadjuvant therapy was statistically significant short than others( $\mathrm{p}=0.02,24$ months DFS; \%31.2 vs \%80.5). This finding maybe reflective of locally advanced disease, but also specially inTNBC may suggest that neoadjuvant chemotherapy may have limited effect and delaying of the surgical treatment may affect the prognosis.

A total of 11 patients were found to be ALDH1-positive and this positivity doesn't seem to be statistically significant alone. ALDH1as a biomarker to identify the breast cancer stem cell groups that can lead to targeted therapies in breast cancer was not found to be a prognostic value alone. Further research combining with other biomarkers and with a greater number of patients is necessary to confirm the role of ALDH1 in TNBC.

\section{Acknowledgements: None.}

Financial Support: This research received no specific grant from any funding agency, commercial or not-for-profit sectors

Conflict of Interest: The authors declared that they had no conflicts of interest.

\section{References}

1. Goldhirsch A, Wood WC, Coates AS, Gelber RD, Thurlimann B, Senn HJ, et al. Strategies for subtypes-dealing with the diversity of breast cancer: highlights of the St. Gallen International Expert Consensus on the Primary Therapy of Early Breast Cancer 2011. Annals of oncology : official journal of the European Society for Medical Oncology / ESMO. 2011;22(8):1736-47.

2. Carey LA, Perou CM, Livasy CA, Dressler LG, Cowan D, Conway $\mathrm{K}$, et al. Race, breast cancer subtypes, and survival in the Carolina Breast Cancer Study. Jama. 2006;295(21):2492-502.

3. Bauer KR, Brown M, Cress RD, Parise CA, Caggiano V. Descriptive analysis of estrogen receptor (ER)-negative, progesterone receptor (PR)-negative, and HER2-negative invasive breast cancer, the so-called triple-negative phenotype: a population-based study from the California cancer Registry. Cancer. 2007;109(9):1721-8.

4. Morris GJ, Naidu S, Topham AK, Guiles F, Xu Y, McCue $\mathrm{P}$, et al. Differences in breast carcinoma characteristics in newly diagnosed African-American and Caucasian patients: a single-institution compilation compared with the National Cancer Institute's Surveillance, Epidemiology, and End Results database. Cancer. 2007;110(4):876-84
5. Stead LA, Lash TL, Sobieraj JE, Chi DD, Westrup JL, Charlot $\mathrm{M}$, et al. Triple-negative breast cancers are increased in black women regardless of age or body mass index. Breast cancer research : BCR. 2009;11(2):R18.

6. Lund MJ, Trivers KF, Porter PL, Coates RJ, LeylandJones $\mathrm{B}$, Brawley $\mathrm{OW}$, et al. Race and triple negative threats to breast cancer survival: a population-based study in Atlanta, GA. Breast cancer research and treatment. 2009;113(2):357-70

7. Metzger-Filho O, Tutt A, de Azambuja E, Saini KS, Viale $\mathrm{G}$, Loi S, et al. Dissecting the heterogeneity of triplenegative breast cancer. Journal of clinical oncology : official journal of the American Society of Clinical Oncology. 2012;30(15):1879-87.

8. Cleator S, Heller W, Coombes RC. Triple-negative breast cancer: therapeutic options. The Lancet Oncology. $2007 ; 8(3): 235-44$.

9. Cianfrocca M, Goldstein LJ. Prognostic and predictive factors in early-stage breast cancer. The oncologist. 2004;9(6):606-16.

10. Kim K, Lee E, Lee J. Clinicopathologic Signature of TNBC Patients with Good Prognosis. Paper presented at: San Antonio Breast Cancer Symposium (SABCS); San Antonio, Texas; Korea Breast Cancer Society. 2009;4065.

11. Millikan RC, Newman B, Tse CK, Moorman PG, Conway $\mathrm{K}$, Dressler LG, et al. Epidemiology of basal-like breast cancer. Breast cancer research and treatment. 2008;109(1):123-39

12. Dent R, Trudeau M, Pritchard KI, Hanna WM, Kahn HK, Sawka CA, et al. Triple-negative breast cancer: clinical features and patterns of recurrence. Clinical cancer research : an official journal of the American Association for Cancer Research. 2007;13(15 Pt 1):4429-34.

13. Collett K, Stefansson IM, Eide J, Braaten A, Wang H, Eide GE, et al. A basal epithelial phenotype is more frequent in interval breast cancers compared with screen detected tumors. Cancer epidemiology, biomarkers \& prevention : a publication of the American Association for Cancer Research, cosponsored by the American Society of Preventive Oncology. 2005;14(5):1108-12.

14. Yang WT, Dryden M, Broglio K, Gilcrease M, Dawood $\mathrm{S}$, Dempsey PJ, et al. Mammographic features of triple receptor-negative primary breast cancers in young premenopausal women. Breast cancer research and treatment. 2008;111(3):405-10.

15. Blows FM, Driver KE, Schmidt MK, Broeks A, van Leeuwen FE, Wesseling J, et al. Subtyping of breast cancer by immunohistochemistry to investigate a relationship between subtype and short and long term survival: a collaborative analysis of data for 10,159 cases from 12 studies. PLoS medicine. 2010;7(5):e1000279.

16. Jackson B, Brocker C, Thompson DC, Black W, Vasiliou $\mathrm{K}$, Nebert DW, et al. Update on the aldehyde dehydrogenase gene (ALDH) superfamily. Human genomics. 2011;5(4):283-303

17. de Beca FF, Caetano P, Gerhard R, Alvarenga CA, Gomes $\mathrm{M}$, Paredes J, et al. Cancer stem cells markers CD44, CD24 and ALDH1 in breast cancer special histological types. Journal of clinical pathology. 2013;66(3):187-91 
18. Gluz O, Nitz UA, Harbeck N, Ting E, Kates R, Herr A, et al. Triple-negative high-risk breast cancer derives particular benefit from dose intensification of adjuvant chemotherapy: results of WSG AM-01 trial. Annals of oncology : official journal of the European Society for Medical Oncology / ESMO. 2008;19(5):861-70.

19. Meyers MO, Klauber-Demore N, Ollila DW, Amos KD, Moore DT, Drobish AA, et al. Impact of breast cancer molecular subtypes on locoregional recurrence in patients treated with neoadjuvant chemotherapy for locally advanced breast cancer. Annals of surgical oncology. 2011;18(10):2851-7
20. Zaky SS, Lund M, May KA, Godette KD, Beitler JJ, Holmes LR, et al. The negative effect of triple-negative breast cancer on outcome after breast-conserving therapy. Annals of surgical oncology. 2011;18(10):2858-65.

21. Mansour EG, Ravdin PM, Dressler L. Prognostic factors in early breast carcinoma. Cancer. 1994;74(1 Suppl):381400 .

22. Liu S, Wicha MS. Targeting breast cancer stem cells. Journal of clinical oncology : official journal of the American Society of Clinical Oncology. 2010;28(25):4006-12.

Copyright (C) 2014 The Author(s); This is an open-access article distributed under the terms of the Creative Commons Attribution License (http://creativecommons.org/licenses/by/4.0), which permits unrestricted use, distribution, and reproduction in any medium, provided the original work is properly cited. 